\title{
Pseudoparasitism by Spalangia cameroni (Hymenoptera: Pteromalidae) of pupae of Ceratitis capitata (Diptera: Tephritidae): Frequency and implications
}

\author{
Luis DE PEDRO ${ }^{1,2}$, Francisco BEITIA ${ }^{2}$, Josep D. ASíS ${ }^{1}$ and José TORMOS ${ }^{1}$ \\ ${ }_{1}^{1}$ Área de Zoología, Facultad de Biología, Universidad de Salamanca, 37007-Salamanca, Spain; \\ e-mails: Idepedro@usal.es, tormos@usal.es, asis@usal.es \\ ${ }^{2}$ Instituto Valenciano de Investigaciones Agrarias, Unidad Asociada de Entomología IVIA/CIB-CSIC, Apartado Oficial, \\ 46113-Montcada, Valencia, Spain; e-mail: beitia_fra@gva.es
} Key words. Hymenoptera, Pteromalidae, Spalangia cameroni, Diptera, Tephritidae, Ceratitis capitata, Mediterranean fruit fly,
parasitoid, pseudoparasitism, overstinging, biological control

\begin{abstract}
The effectiveness of natural enemies in controlling pests may be determined by many traits linked to their ability to regulate the density of their prey. In this respect, the phenomenon of pseudoparasitism, in which female parasitoids reject a host after inserting their ovipositor into it, is fairly common among hymenopteran parasitoids. However, in spite of this its effect on hosts is rarely reported in entomological and biological control literature. For this reason, in the present study, the pseudoparasitism by the parasitoid Spalangia cameroni Perkins of the Mediterranean pest Ceratitis capitata (Wiedemann) and its effect on several biological parameters of the host were studied under laboratory conditions. The results indicate that the percentage pseudoparasitism by S. cameroni of medfly in the laboratory is high, even slightly higher than host-feeding, which is commonly used to evaluate the potential of parasitoids as biological control agents. In addition, the adults that emerge from pseudoparasitized medfly pupae have a male-biased sex ratio, low levels of survival and are frequently damaged, which results in small adults and an inability to mate successfully. In conclusion, our results indicate that pseudoparasitism is common and enhances the effectiveness of $S$. cameroni attacking medfly, which highlights the importance of this phenomenon when selecting parasitoids to be included in a biological control programme.
\end{abstract}

\section{INTRODUCTION}

The Mediterranean fruit fly, Ceratitis capitata (Wiedemann) (Diptera: Tephritidae), also known as the medfly, is currently an important pest of the Spanish citrus industry. It is a highly polyphagous and multivoltine species, which has a high fecundity and is globally widespread (Fletcher, 1989; Liquido et al., 1991; Papadopoulos, 2008). Thus, this species is subject to quarantine measures in many countries (EPPO, 2012), and special control measures are needed in areas affected by this pest, such as the biological control of this species using native natural enemies in recent years.

In this regard, Spalangia cameroni Perkins (Hymenoptera: Pteromalidae) is an idiobiont and solitary pupal ectoparasitoid widely used in America, Europe and Australia for the biological control of house and stable flies (King, 1988; Birkemoe et al., 2009; Tormos et al., 2009). This species was first identified as a parasitoid of $C$. capitata in 2006, when it was reported as a native pupal parasitoid of this pest in Spain (Falcó et al., 2006). Thus, since 2006, $S$. cameroni has been bred in the laboratories of the Instituto Valenciano de Investigaciones Agrarias (IVIA, Valencia,
Spain) using medfly pupae (and, since 2009, medfly pupae killed by cold-shock) as hosts, for use in experiments that assess its usefulness as a biological weapon against this pest (Tormos et al., 2010, 2014). In this respect, the effect of natal host and learning on the host preference of $S$. cameroni and the intraguild interactions between this parasitoid and other natural enemies (Tormos et al., 2018a, b) have recently been assessed. In both of these studies the "induced mortality" attributed to parasitoids, which is defined as the mortality of pupae, from which adults do not emerge (de Pedro et al., 2017), was recorded, since it is one of the most relevant parameters regarding the parasitic activity of a parasitoid wasp.

Induced mortality may be caused by several factors, such as oviposition, host-feeding or pseudoparasitism. The term pseudoparasitism, also known as overstinging (Cebolla et al., 2018), was originally coined by Jones (1985) and describes the rejection of a host in which a female parasitoid has inserted its ovipositor without feeding or ovipositing. More precisely, pseudoparasitized hosts may be defined as stung hosts that do not contain living parasites. This 
phenomenon can lead directly to host death (e.g., through mechanical injury caused by ovipositor insertion) or to mutilation, which as a consequence reduces its fecundity, fertility and survival (Brown \& Kainoh, 1992; Biondi et al., 2013; Cebolla et al., 2018); nevertheless, in both cases it increases the effectiveness of the parasitic activity of a parasitoid. However, despite the importance of pseudoparasitism in the performance of parasitoids as biological control agents, this phenomenon and its implications are rarely mentioned in entomological literature.

Therefore, in the present study, the frequency of pseudoparasitism by $S$. cameroni of $C$. capitata, and its effect on several parameters of the host were assessed, under controlled laboratory conditions.

\section{MATERIAL AND METHODS}

\section{Study centre and insects}

All experiments in this study were performed in compliance with current Spanish law. This study was carried out at the facilities of IVIA. The medfly pupae and $S$. cameroni adults used in this study were obtained from standard cultures maintained at IVIA, where both insects have been reared on a large scale for more than eight years. The rearing processes for $C$. capitata and $S$. cameroni are described by Albajes \& Santiago-Álvarez (1980a, b) and Pérez-Hinarejos \& Beitia (2008), respectively. Ceratitis capitata was reared in methacrylate boxes $(40 \times 30 \times$ $30 \mathrm{~cm}$ ), containing sugar and hydrolyzed protein (in a ratio of $4: 1$ ) and water as nutritional sources, and equipped with a front cloth-covered window for egg deposition. Eggs were collected in plastic trays partially filled with water and placed under the rearing cages, before being placed in artificial medfly diet, where they developed into larvae and pupae. The artificial medfly diet was made of wheat bran, sugar, brewer's yeast, methylparaben Nipagin $\AA$, propylparaben Nipasol $\AA$, benzoic acid Panreac ${ }^{\circledR}$ and water.

Spalangia cameroni was reared in small methacrylate cages $(30 \times 20 \times 15 \mathrm{~cm})$ with an upper cloth-covered window for ventilation and water, honey and sugar as sources of nutrition. As hosts, 10, 2-5-day-old live medfly pupae (obtained from medfly stock cultures), were offered to each parasitoid female every 48 $\mathrm{h}$. This number was selected because it resulted in high numbers of parasitized pupae, eggs laid and offspring produced (Tormos et al., 2012). Each rearing cage housed around 600 parasitoids (the sex ratio of the $S$. cameroni adults was $1: 1$ ) and every three days 100 newly emerged individuals (50 females and 50 males) were added to each cage.

To perform the experiments, medfly pupae that were attacked (parasitized, fed on or pseudoparasitized) by S. cameroni females and non-attacked were obtained from the above mentioned cultures of S. cameroni and C. capitata, respectively, between 2006 and 2009. The laboratory conditions for both rearing and experiments were $21-24^{\circ} \mathrm{C}, 55-80 \% \mathrm{RH}$, and a $16 \mathrm{~L}: 8 \mathrm{D}$ photoperiod.

\section{Experimental design}

Two experiments were conducted to assess the frequency of pseudoparasitism by $S$. cameroni (Experiment 1) in the laboratory and the effect thus has on several biological parameters of the host, such as survival, body size and sex ratio (Experiment 2).

Experiment 1. Frequency of pseudoparasitism by S. cameroni in the laboratory
As in a previous study by the same authors on superparasitism by $S$. cameroni of medfly in the laboratory (Tormos et al., 2012), first a method for estimating the frequency of pseudoparasitism was determined. This consisted of determining the degree of pseudoparasitism based on the presence of "marks" on the surface of a puparium. These marks (holes) can be of three types depending on the type of attack. Based on our experience: oviposition holes, are each surrounded by a dark ring that is surrounded by a bleached halo (like a miniature raised crater), which is formed when the ovipositor is withdrawn; host-feeding holes, each have a whitish halo (probably due to drops of fluid produced by the female, which aid the development of oocytes inside eggs) around the puncture; and pseudoparasitism holes, in which the puncture does not have a halo.

To confirm this and supply preliminary data, a random sample of medfly pupae was taken from the laboratory cultures of $S$. cameroni on three occasions (replicates). From this sample, a total of 300 "marked" pupae were separated on each occasion, 100 of each type of mark, in plastic trays $(20 \times 12 \times 3 \mathrm{~cm}$, one for each type of mark and occasion) after determining the type of mark on each host pupa by examining it under a stereomicroscope. When a single pupa had more than one type of mark, it was classified as "parasitized" if there was at least one oviposition hole; in the absence of oviposition holes, pupae were classified as "pseudoparasitized" when there were no host-feeding holes. This determination was confirmed in each case by dissecting these pupae under a microscope, in order to determine whether an egg was present [parasitism (oviposition)] or not (host-feeding/pseudoparasitism). In addition, in the latter case, whether the pupae showed signs that the parasitoid had fed on them, such as the above mentioned whitish halo (host-feeding), or not (pseudoparasitism), was recorded. Furthermore, pupae from the same parasitoid rearing showing no sign of attack were also dissected; no eggs or signs of feeding were recorded on any of them. A Leica MZ9.5 high-performance microscope was used for examining the marks and the dissected pupae; sample preparation and dissection were done using the same procedure, with pupae placed on cavity slides in saline solution in order to obtain a better display of each pupa. After being separated, all pupae were placed in Petri dishes in a climatic chamber at $5^{\circ} \mathrm{C}$ (to prevent egg development) for a few days before dissection, as there was insufficient time to separate and dissect them on the same day.

Thus, in this experiment, the medfly pupae attacked in the laboratory cultures of $S$. cameroni were collected on three occasions (replicates) until a satisfactory number was reached (total: 603). Following the above mentioned procedure, the number of each type of attack was scored, in order to estimate the frequency of pseudoparasitism versus parasitism and host-feeding in laboratory cultures.

\section{Experiment 2. Effect of pseudoparasitism on host fitness and survival}

This experiment was designed to evaluate the effect of pseudoparasitism on several biological parameters of the host, such as survival or body size and sex ratio. For this purpose, the pseudoparasitized pupae obtained in the previous experiment were kept in plastic boxes $(20 \times 15 \times 10 \mathrm{~cm})$ with a cloth-covered window in the lid for ventilation, until the emergence of adults. The number of male and female descendants was scored in order to obtain the survival and offspring sex ratio. Moreover, the body size of male and female descendants was estimated based on the size of their wings (Zucoloto, 1987; Rodriguero et al., 2002). For this, the wings of 20 male and 20 female specimens from each replicate were separated and placed on "wet-mount" microscope slides, in distilled water and covered by a plastic coverslip. Wings 
were measured to the nearest $0.001 \mathrm{~mm}$ using a Leica MZ12.5 high-performance stereomicroscope equipped with a Canon S50 camera and Leica IM50 software (Leica Microsystems Imaging Solutions). Wing length was measured as the distance between the intersection of the humeral $(\mathrm{H})$ and costal $(\mathrm{C})$ veins and the apex of the radial vein $\left(\mathrm{R}_{4+5}\right)$ (Rodriguero et al., 2002).

To assess the effect of pseudoparasitism on the above mentioned biological parameters, survival, body size and sex-ratio, results obtained for pseudoparasitized medfly pupae were compared with those for the same number of non-attacked pupae, randomly separated from the medfly culture and, consequently, isolated from parasitoids.

\section{Statistical analysis}

As mentioned above, each type of attack (parasitism, hostfeeding and pseudoparasitism) and its corresponding frequency was determined based on the type of mark observed. The relationship among the frequencies of the different types of attack was analysed using a Pearson's chi-squared $\left(\chi^{2}\right)$ test. This test was also used to determine whether the survival and offspring sex ratio of pseudoparasitized and non-attacked pupae differed significantly. Moreover, two-way factorial ANOVA was used to test body size depending on the type of pupae (pseudoparasitized vs. non-attacked), with occasion (block effect) a random factor.

Prior to the analysis, data normality was checked and data transformed when necessary. Values are reported as absolute numbers or percentages (\%). Data were analyzed using the IBM SPSS 17.0 statistical software package (IBM SPSS, Chicago, IL, USA), with $\alpha=0.05$.

\section{RESULTS}

\section{Experiment 1. Frequency of pseudoparasitism in laboratory cultures}

Of the 603 attacked pupae, 279 (46.2\%) had oviposition holes, 156 (25.8\%) host-feeding holes and 168 (27.9\%) only pseudoparasitism holes (Table 1). There were significant differences among the frequencies of the three types of attack $\left(\chi^{2}=21.298\right.$, d.f. $\left.=2, P \leq 0.0001\right)$. Nevertheless, these differences were due to more of the attacked pupae being parasitized rather than used for host-feeding or pseu-

Table 1. Pupae with different types of "marks" (oviposition holes: 279; host-feeding holes: 156; pseudoparasitism holes: 168) obtained from a random sample of attacked medfly pupae, on several occasions, from laboratory cultures of $S$. cameroni. The standardized residuals obtained from the chi-squared test only show statistically significant differences for parasitism at $95 \%$ level.

\begin{tabular}{lccc}
\hline Type of attack & $\begin{array}{c}\text { Attacked } \\
\text { pupae }\end{array}$ & $\begin{array}{c}\text { Attacked } \\
\text { pupae }(\%)\end{array}$ & $\begin{array}{c}\text { Standardized } \\
\text { residuals }\left(\mathrm{X}^{2}\right)\end{array}$ \\
\hline Parasitism & 279 & 46.2 & 2.5 \\
Host-feeding & 156 & 25.8 & -1.7 \\
Pseudoparasitism & 168 & 27.9 & -1.2 \\
\hline
\end{tabular}

doparasitized (see Table 1), since the incidence of the latter two behavioural traits were not statistically significantly different; however, it should be noted that pseudoparasitism occurred more frequently than host-feeding (Table 1).

\section{Experiment 2. Effect of pseudoparasitism on host fitness and survival}

Adult hosts only emerged from 117 of the 168 pseudoparasitized pupae $(69.6 \%)$ and had a male-biased sex ratio (74.3\%). A chi-squared test revealed significant differences between pseudoparasitized and non-attacked pupae in terms of survival, measured as the number of emergences $\left(\chi^{2}=\right.$ 45.343 , d.f. $=1, \mathrm{P}<0.001)$. A significantly higher number of pseudoparasitized pupae died and, consequently, fewer adults emerged than from non-attacked pupae. Moreover, a significant effect of the type of pupae (pseudoparasitized/ non-attacked) on the host sex ratio $\left(\chi^{2}=7.739, \mathrm{df}=1, \mathrm{P}=\right.$ $0.005)$ was also recorded. In this regard, adults emerging from pseudoparasitized pupae, unlike non-attacked pupae $\left(\chi^{2}=0.191\right.$, d.f. $\left.=1, P=0.662\right)$, had a male-biased sex ratio $\left(\chi^{2}=10.319\right.$, d.f. $\left.=1, \mathrm{P}=0.002\right)$. In addition, a two-way factorial ANOVA $\left[\sigma^{2}\right.$ (residual variance): $301.38,{ }_{\sigma B}^{2}$ (block variance): $5.6 \times 10^{-4}$ ] revealed that males emerging from pseudoparasitized pupae were significantly smaller than females $\left(\mathrm{F}_{1,152}=34.057, \mathrm{P}=0.01\right)$ and also smaller than males that emerged from non-attacked pupae $\left[\sigma^{2}\right.$ (residual variance): $590.12,{ }_{\sigma B}^{2}$ (block variance): $\left.4.8 \times 10^{-3}\right]\left(\mathrm{F}_{1,152}=\right.$ 837.138, $\mathrm{P}<0.001)$.

\section{DISCUSSION}

The results of this study indicate that in laboratory cultures of $S$. cameroni, pseudoparasitism (i.e., the rejection of medfly pupae by the females of this parasitoid, without ovipositing or host-feeding, after stinging them with their ovipositor), is a common behavioural trait, as it is in other hymenopteran parasitoids, both under controlled conditions and in the wild (Jones et al., 1986; Hegazi \& Khafagi, 2000; Cebolla et al., 2018). In addition, the results indicate that in $S$. cameroni this behaviour is more frequent when attacking male pupae and can result in the death of the host or, in many cases, damage that results in smaller adult hosts.

Regarding the host mortality linked to pseudoparasitism, it should be noted that it represents a case of "overkill", since in this case hosts are not used for feeding or ovipositing. In previous studies (Legner \& Gerling, 1967; Gerling \& Legner, 1968) it is reported that many S. cameroni females feed on the first host they encounter and, upon completion of host-feeding, begin to search for a suitable ovi-

Table 2. Survival, sex ratio and body size of adult medflies that emerged from pseudoparasitized and non-attacked pupae ( $\mathrm{n}=168$ in both cases). These pupae were obtained from laboratory cultures on three occasions. In each column, values with different lower case letters differ significantly at the $95 \%$ level.

\begin{tabular}{lccc}
\hline Type of pupae & $\begin{array}{c}\text { Survival (Emergences, } \\
\text { Deaths; \%) }\end{array}$ & $\begin{array}{c}\text { Sex ratio } \\
(\delta,+; \delta / \delta+\%)\end{array}$ & $\begin{array}{c}\text { Male size (Range; } \\
\text { Mean } \pm \text { SE) }(\mathrm{mm})\end{array}$ \\
\hline Pseudoparasitized $^{1}$ & 117,$51 ; 69.6 \mathrm{a}$ & 87,$30 ; 0.74 \mathrm{a}$ & $3.4-3.8 ; 3.6 \pm 1.14 \mathrm{a}$ \\
Non-attacked $^{2}$ & 163,$5 ; 97.1 \mathrm{~b}$ & 78,$85 ; 0.48 \mathrm{~b}$ & $3.7-4.3 ; 3.9 \pm 0.18 \mathrm{~b}$ \\
\hline
\end{tabular}

${ }^{1}$ Pupae randomly collected from the $S$. cameroni culture and determined as pseudoparasitized in Experiment $1 .{ }^{2}$ Pupae randomly collected from the medfly culture. 
position site on a second puparium. These studies do not refer to "overkilling" ability; however, in our study, this parasitoid killed more hosts than needed for parasitism or host-feeding. This type of host-killing is reported in other parasitoids (Jones et al., 1986; Abram et al., 2016) and is a desirable trait when assessing their potential as biocontrol agents, as it is for predators (Johnson et al., 1975).

The other most significant consequence of $S$. cameroni pseudoparasitism of medfly is the effect it has on the size and sex ratio of the host offspring. In this regard, our study revealed that pseudoparasitized pupae produced male-biased offspring. According to previous studies (King, 2002; Beitia et al., 2016), this could be due to the smaller size of male medfly pupae, making them less beneficial or attractive to ovipositing females of $S$. cameroni and thus being more frequently rejected than female pupae. Furthermore, the male descendants were smaller in size than females and also than male offspring produced by non-attacked pupae. On this point, it should be noted that in medfly, unlike in other tephritid species such as Rhagoletis cerasi (Linnaeus) and Bactrocera dorsalis (Hendel) (Boller et al., 1977; Iga, 1981), body size is one of the most important factors determining mating success. More specifically, mating occurs more often in this species when males are of equal size or larger than the females (Churchill-Stanland et al., 1986; Krainacker et al., 1989; Bloem et al., 1993; Orozco \& López, 1993; Rodriguero et al., 2002). This could be due to the fact that large males have large wings and thus are able to displace larger air masses that produce louder sounds, which may be attractive for females (Churchill-Stanland et al., 1986). Therefore, the negative effect of pseudoparasitism on the size of the host adversely affects mating in the next generation of $C$. capitata and thus could reduce medfly population increase, which is one of the main goals of every control programme.

In summary, on the basis of the results of the present study it can be concluded that, since pseudoparasitism is common in hymenopteran parasitoids (Cebolla et al., 2018) and adversely affects the biotic potential of its host (in this case, C. capitata), it should be considered as a relevant beneficial trait and taken into account when selecting a parasitoid to be included in a biological control programme. Furthermore, the results of this study increase our knowledge of the biology and parasitic ability of $S$. cameroni when attacking medfly pupae and adds new results to those of recent studies (Beitia et al., 2016; Tormos et al., 2018a,b) on its efficiency as a biological control agent against this important pest.

ACKNOWLEDGEMENTS. This study was carried out in the Centro de Protección Vegetal y Biotecnología, at the IVIA (Valencia, Spain). Funding: This work was partially supported by the Ministerio de Ciencia e Innovación of Spain (MICINN; projects AGL2010-21349-C02-02 and CGL2010-16730), and the Consellería de Agricultura, Pesca y Alimentación (Generalitat Valenciana, Spain). L. de Pedro was funded by an FPU grant (Programa Nacional Español de Becas de Formación de Profesorado Universitario; grant reference: AP2010-2340). Authors confirm that there is no conflict of interests.

\section{REFERENCES}

Abram P.K., Brodeur J., Burte V. \& Boivin G. 2016: Parasitoidinduced host egg abortion: an underappreciated component of biological control services provided by egg parasitoids. Biol. Contr. 98: 52-60.

Albajes R. \& Santiago-Álvarez C. 1980a: Efectos de la densidad larvaria y de la alimentación en la proporción de sexos de Ceratitis capitata (Diptera, Trypetidae). - An. Inst. Nac. Invest. Agr. (Ser. Agríc.) 13: 175-182.

Albajes R. \& Santiago-Álvarez C. 1980b: Influencia de la temperatura en el desarrollo de Ceratitis capitata (Diptera, Trypetidae). - An. Inst. Nac. Invest. Agr. (Ser. Agríc.) 13: 183-190.

Beitia F., Valencia E., Peris B., de Pedro L., Asís J.D. \& TorMOS J. 2016: Influence of natal host on parasitism by Spalangia cameroni (Hymenoptera: Pteromalidae). - Eur. J. Entomol. 113: 99-103.

Biondi A., Desneux N., Amiens-Desneux E., Siscaro G. \& ZapPALÀ L. 2013: Biology and developmental strategies of the palaearctic parasitoid Bracon nigricans on the neotropical moth Tuta absoluta. - J. Econ. Entomol. 106: 1638-1647.

Birkemoe T., Soleng A. \& AaK A. 2009: Biological control of Musca domestica and Stomoxys calcitrans by mass releases of the parasitoid Spalangia cameroni on two Norwedian pig farms. - BioControl 54: 425-436.

Bloem K., Bloem S., Chambers D. \& Muniz E. 1993: Field evaluation of quality: release-recapture of sterile medflies of different sizes. In Aluja M. \& Liedo P. (eds): Fruit Flies: Biology and Management. Springer, New York, pp. 295-296.

Boller E.F., Remund U. \& Katsoyannos B. 1977: Quality control in European cherry fruit fly: evaluation of mating activity in laboratory and field-cage tests. - J. Appl. Entomol. 83: 183-201.

Brown J.J. \& KaINOH Y. 1992: Host castration by Ascogaster sp. (Hymenoptera: Braconidae). - Ann. Entomol. Soc. Am. 85: 67-71.

Cebolla R., Vanaclocha P., Urbaneja A. \& Tena A. 2018: Overstinging by hymenopteran parasitoids causes mutilation and surplus killing of hosts. - J. Pest Sci. 91: 327-339.

Churchill-Stanland C., Stanland R., Wong T.T.I., Tanaka N., McInNIS D.O. \& Dowell R.V. 1986: Size as a factor in the mating propensity of Mediterranean fruit flies, Ceratitis capitata (Diptera: Tephritidae), in the laboratory. - J. Econ. Entomol. 79: 614-619.

de Pedro L., Beitia F., Ferrara F., Asís J.D., Sabater-Muñoz B. \& Tormos J. 2017: Effect of host density and location on the percentage parasitism, fertility and induced mortality of Aganaspis daci (Hymenoptera: Figitidae), a parasitoid of Ceratitis capitata (Diptera: Tephritidae). - Crop Prot. 92: 160-167.

EPPO 2012: PQR - EPPO Database on Quarantine Pests. URL: http://www.eppo.int (last accessed 16 Feb. 2018).

Falcó J.V., Garzón-Luque E., Pérez-Hinarejos M., Tarazona I., Malagón J. \& Beitia F. 2006: Two native pupal parasitoids of Ceratitis capitata (Diptera, Tephritidae) found in Spain. IOBC/WPRS Bull. 29: 71-74.

FLETCHER B.S. 1989: Life history strategies of tephritid fruit flies. In Robinson S. \& Hooper G. (eds): Fruit Flies: Their Biology, Natural Enemies and Control. Elsevier, Amsterdam, pp. 195209.

GerLing D. \& Legner E.F. 1968: Developmental history and reproduction of Spalangia cameroni, parasite of synanthropic flies. - Ann. Entomol. Soc. Am. 61: 1436-1443.

Hegazi E.M. \& Khafagi W.E. 2000: Possible bases of pseudoparasitism in Spodoptera littoralis larvae stung by Microplitis rufiventris. - J. Insect Physiol. 46: 1267-1274. 
IGA M. 1981: Mating preference of Dacus dorsalis (Hendel) (Diptera: Tephritidae) with reference to individual variations in size. - J. Appl. Entomol. 25: 292-294.

Johnson D., Akre B.G. \& Crowley P.H. 1975: Modeling arthropod predation: wasteful killing by damselfly naiads. - Ecology 56: 1081-1093.

JONES D. 1985: Parasite regulation of host insect metamorphosis: a new form of regulation in pseudoparasitized larvae of Trichoplusia ni. - J. Comp. Physiol. 155: 583-590.

Jones D., Jones G., Rudnicka M., Click A., Reck-Malleczewen V. \& IwaYa M. 1986: Pseudoparasitism of host Trichoplusia $n i$ by Chelonus spp. as a new model system for parasite regulation of host physiology. - J. Insect Physiol. 32: 315-328.

King B.H. 1988: Sex-ratio manipulation in response to host size by the parasitoid wasp Spalangia cameroni: a laboratory study. - Evolution 42: 1190-1198.

KING B.H. 2002: Offspring sex ratio and number in response to proportion of host sizes and ages in the parasitoid wasp Spalangia cameroni (Hymenoptera: Pteromalidae). — Environ. Entomol. 31: 505-508.

Krainacker D.A., CARey J.R. \& Vargas R.I. 1989: Size-specific survival and fecundity for laboratory strains of two tephritids (Diptera: Tephritidae) species: implications for mass rearing. - J. Econ. Entomol. 82: 104-108.

Legner E.F. \& Gerling D. 1967: Host-feeding and oviposition on Musca domestica by Spalangia cameroni, Nasonia vitripennis, and Muscidifurax raptor (Hymenoptera: Pteromalidae) influences their longevity and fecundity. - Ann. Entomol. Soc. Am. 60: $678-691$.

Liquido N.J., ShindoA L.A. \& CunNingham R.T. 1991: Host Plants of the Mediterranean Fruit Fly, C. capitata (Wiedemann) (Diptera: Tephritidae): An Annotated World Review. Miscellaneous Publication 77. Entomological Society of America, Lanham, MD, $52 \mathrm{pp}$.

Orozco D. \& LÓPEZ R.O. 1993: Mating competitiveness of wild and laboratory mass-reared medflies: effect of male size. In Aluja M. \& Liedo P. (eds): Fruit Flies: Biology and Management. Springer, New York, pp. 185-188.

Papadopoulos N.T. 2008: Mediterranean fruit fly, Ceratitis capitata (Wiedemann) (Diptera: Tephritidae). In Capinera J.L. (ed.): Encyclopaedia of Entomology. Vol. 3. Springer, Heidelberg, pp. 2318-2322.

Pérez-Hinarejos M. \& Beitia F. 2008: Parasitism of Spalangia cameroni (Hymenoptera, Pteromalidae), an idiobiont parasitoid of Ceratitis capitata (Diptera, Tephritidae). - IOBC/ WPRS Bull. 38: 130-133.

Rodriguero M.S., Vilardi J.C., Vera M.T., Cayol J.P. \& Rial E. 2002: Morphometric traits and sexual selection in medfly (Diptera: Tephritidae) under field cage conditions. - Fla Entomol. 85: 143-149.

Tormos J., Beitia F., Boeckmann E.A. \& Asís J.D. 2009: The preimaginal stages and development of Spalangia cameroni Perkins (Hymenoptera: Pteromalidae) on Ceratitis capitata (Wiedemann) (Diptera: Tephritidae). - Micron 40: 646-658.

Tormos J., Beitia F., Alonso M., Asís J.D. \& Gayubo S. 2010: Assessment of Ceratitis capitata (Diptera: Tephritidae) pupae killed by heat or cold as hosts for rearing Spalangia cameroni (Hymenoptera: Pteromalidae). — Ann. Appl. Biol. 16: 179185.

Tormos J., Asís J.D., Sabater-Muñoz B., Baños L., Gayubo S.F. \& Beitia F. 2012: Superparasitism in laboratory rearing of Spalangia cameroni (Hymenoptera: Pteromalidae) a parasitoid of medfly (Diptera: Tephritidae). — Bull. Entomol. Res. 102: $51-61$.

Tormos J., Sabater-Muñoz B., Asís J.D. \& Beitia F. 2014: Validation of a methodology for rearing Spalangia cameroni (Hymenoptera: Pteromalidae) on Ceratitis capitata (Diptera: Tephritidae). - Can. Entomol. 146: 676-683.

Tormos J., Beitia F., Asís J.D. \& DE Pedro L. 2018a: Natal host and learning as factors in host preference by Spalangia cameroni Perkins (Hymenoptera: Pteromalidae). - Crop Prot. 110: $155-159$.

Tormos J., Beitia F., Asís J.D. \& de Pedro L. 2018b: Intraguild interactions between two biological control agents in citrus fruit: implications for biological control of medfly. - Ann. Appl. Biol. 172: 321-331.

Zucoloto F.S. 1987: Feeding habits of Ceratitis capitata: Can larvae recognize a nutritional effective diet? - J. Insect Physiol. 33: 349-353.

Received April 20, 2018; revised and accepted July 20, 2018 Published online August 29, 2018 